\title{
Mujer Gitana y Educación: un Camino hacia los Derechos Humanos
}

\author{
Ana Ayuste González y Montserrat Payà Sánchez
}

Universidad de Barcelona, España

\begin{abstract}
The existence of four world-wide conferences, declarations, conventions and proposals of action to study and face up to women's unequal situation, as far as human rights are concerned, is an indicator of a wide politically-based social change. This social change must be accompanied, though, by institutional changes, law modifications, consistent educational practices and respectful quotidian attitudes if we really want it to be genuine. This paper presents the results from an investigation carried out in three counties in Spain, during the period 2000-2003, which was aimed to discover the factors that impede gipsy girls and teenagers to finish their schooling. The investigation has been carried out in context of human rights, women, minorities and education. Key words: Gipsy women, minorities, schooling, discrimination, equality of opportunities.
\end{abstract}

\section{RESUMEN}

La existencia de cuatro conferencias mundiales, declaraciones, convenciones y propuestas de acción destinadas a estudiar y enfrentar la situación desigual de las mujeres ante los derechos humanos, es un indicador de cambio social profundo, de carácter político, pero que, sin embargo, debe ir acompañado de cambios institucionales, modificaciones en las legislaciones, prácticas educativas consecuentes y actitudes cotidianas respetuosas, si se pretende que se haga realidad. El artículo presenta los resultados de una investigación llevada a cabo en tres Comunidades Autónomas del Estado Español en el período 2000-03, orientada a descubrir los factores que dificultan que las niñas y adolescentes de la comunidad gitana continúen con su escolaridad, dentro del contexto derechos humanos, mujer, minorías y educación.

Descriptores: Mujer gitana, minorías, escolarización, discriminación, igualdad de oportunidades.

RÉSUMÉ

L'existence de quatre conférences mondiales, déclarations, conventions et propositions d'actions destinées à étudier et confronter la situation d'inégalité des femmes face aux Droits de l'Homme, est un indicateur de profond changement social, de caractère politique, mais qui, pourtant doit être accompagné de changements institutionnels, modifications des législations, pratiques éducatives conséquentes et attitudes quotidiennes respectueuses, si on prétend qu'il se fasse réalité. Cet article présente les résultats d'une recherche réalisée dans trois Régions Autonomes de l'Etat Espagnol sur la période 2000-2003, destinée à découvrir les facteurs qui empêchent que les filles et adolescentes de la Communauté Gitane puissent continuer leur scolarité dans le contexte des Droits de l'Homme, de la Femme, des minorités et de l'Education.

Mots-cléfs: Femme gitane, monorités, scolarité, discrimination, l'égalité des chances. 
Entre nosotros existen a todas luces diferencias muy reales en cuanto a la raza, la edad y el sexo. Mas no son esas diferencias las que nos separan. Lo que nos separa es, por el contrario, nuestra negativa a reconocer las diferencias y a analizar las distorsiones que derivan de darles nombres falsos tanto a ellas como a sus efectos en la conducta y en las expectativas humanas (Audre Lorde, 2002, 123).

\section{Introducción}

$\mathrm{E}$ L Ámbito De Los rDerechos Humanos se relaciona con valores tales como dignidad, unidad, justicia, igualdad y equidad. Tras la $2^{\text {a }}$ Guerra Mundial y motivado por el reconocimiento de que las relaciones internacionales y sociales no podían volver a reproducir la hecatombe de la $2^{a}$ gran guerra, se aventuraba un futuro de relación basado en la unidad y la igualdad que culminaba con la creación de la ONU en 1945, substituyendo a la antigua Sociedad de Naciones que se había revelado como ineficaz al no poder evitar el conflicto bélico, y la aprobación de la Declaración Universal de Derechos Humanos en 1948. El primer escollo que se presentó en aquel entonces y que continúa vigente en nuestros días, fue el de no conferir a la Declaración el carácter prescriptivo de ley que obligara a su cumplimiento, quedando reservada en un plano ciertamente ideal y de meta a alcanzar. Desde entonces, indudablemente, se ha avanzado mucho en los planos ideal, legislativo y de actuación. Sin embargo, la realidad actual, a nivel mundial y a nivel cotidiano también, sigue surtiendo de hechos que atentan contra la dignidad debida a todo ser humano: se ha avanzado, pero queda todavía mucho camino por recorrer.

La enseñanza de los Derechos Humanos es una área o eje transversal presente en los diseños curriculares básicos de muchos países y constituye, sin duda, una vía muy válida para generar cambios favorables en las nuevas generaciones que estarán, así, mejor preparadas para el ejercicio de la ciudadanía. Pero antes, habría que asegurar el derecho de todos los niños y niñas a la educación, una educación que tiene que ser la mejor de las posibles y sustentada en el pilar de la equidad. No se puede pensar en una educación igualitaria, sino en una educación que contribuya a superar posibles barreras y condicionantes desfavorables presentes ya en el momento del nacimiento e, incluso, antes. Desde esta perspectiva, las niñas continúan siendo un colectivo en desventaja simplemente por su género. La mayoría de personas analfabetas en el mundo son mujeres. Las mujeres también son, juntamente con la infancia, la mayor parte de las víctimas de la violencia. La violación, a mujeres y niñas, ha sido considerada por el Tribunal Penal Internacional como arma de guerra y de genocidio. En igualdad de empleo, la retribución económica suele ser menor para las mujeres que para los hombres. ¿Cuántas mujeres acceden a jefes de estado? ¿Qué proporción de mujeres ocupa cargos directivos y de responsabilidad en el ámbito de la empresa o la función pública? ¿Cuándo apareció el concepto de "discriminación positiva”, aplicado especialmente en el ámbito de la política? ¿A cuántas mujeres identificarían nuestros/as estudiantes de Primaria y Secundaria como relevantes en el plano histórico, científico, literario, artístico, etc.? Los estereotipos sociales siguen cebándose en el colectivo femenino... y así podríamos continuar en relación a múltiples aspectos. La realización, cada vez más plena, de los derechos humanos pasa, segura- 
mente, por asegurar el derecho de las niñas y mujeres a la educación. Ésta sería la antesala de la realización de otros muchos derechos tanto para hombres como para mujeres. Y desde esta perspectiva todas las acciones reales que contribuyan a superar la situación desfavorable y de exclusión que viven las niñas en lugares distintos en este mismo momento, pueden ser consideradas urgentes y prioritarias.

\section{El Derecho de la Mujer a la Educación}

Reclamar el derecho de la mujer a la educación puede parecer algo incluso innecesario. ¿Acaso no está recogido en el artículo 26 de la Declaración Universal de los Derechos Humanos de 1948? A nivel ideal, de formulación de principios, sí que parece una reclamación redundante, pero la realidad, la práctica de tal derecho, es otra muy distinta, y la mujer, por el hecho de serlo, se enfrenta a obstáculos en su acceso a la educación que niegan precisamente el artículo primero de la Declaración: "Todos los seres humanos nacen iguales y libres en dignidad y derechos". Es largo y también fructífero el camino que las mujeres han recorrido y recorren por conseguir la igualdad de derechos, desde la Revolución Francesa de 1789, cuando por vez primera las mujeres reclamaron su derecho a voto, hasta nuestros días. Poco a poco, esta cuestión ha ido cobrando visibilidad y haciendo que el grueso de la sociedad se fuera sensibilizando, también paulatinamente. Desde este planteamiento, la ONU ha celebrado cuatro Conferencias Mundiales sobre la Mujer. En la primera, celebrada en México en el 1975, se acordó proclamar el "Decenio de las Naciones Unidas para la Mujer" (1975-1985), como componente prioritario de un plan de acción. En el 1979, y firmada por 130 países, la Asamblea General de la ONU aprobaba la "Convención sobre la Eliminación de Todas las Formas de Discriminación contra la Mujer", documento clave en la lucha por el reconocimiento y la igualdad, porque va más allá de la aparente igualdad formal entre hombres y mujeres para apostar por la igualdad de oportunidades y resultados. El artículo 10 de la "Convención" hace referencia especial a la educación y se dirige tanto hacia el cambio actitudinal de hombres y mujeres, como a reducir la tasa de abandono de los estudios de las niñas, jóvenes y mujeres. De alguna manera, se considera que:

(...) la igualdad en educación constituye el fundamento de plena realización de todos los derechos de la mujer en todas las esferas: en el lugar de trabajo, en la familia y en la sociedad en general.

Puede, por lo tanto, ser considerado el "principal instrumento internacional de derechos humanos de las mujeres" especialmente desde que en 1999 -altamente significativos los veinte años transcurridos entre su aprobación y la implantación de la siguiente medida- el Protocolo Facultativo estableciera dos vías o procedimientos para hacer factibles sus premisas: un mecanismo de investigación de violaciones sistemáticas y/o graves a los derechos humanos de las mujeres, y otro de denuncia de violaciones de la "Convención".

En 1980, en Dinamarca, se celebraba la II Conferencia Mundial sobre la Mujer, en la que se evaluó el desarrollo del "Decenio" y se aprobaron, con el fin de optimizarlo, propuestas de acción para la segunda parte del mismo, centradas especialmente en los 
ámbitos de salud, educación y empleo. La III Conferencia tuvo lugar en Kenia, el 1985. Además de evaluar los procesos, logros y dificultades encontradas durante el desarrollo del "Decenio", en ella se aprobó por consenso un documento ${ }^{2}$ en el que se proponían una serie de medidas -a nivel regional, nacional e internacional-, dirigidas a estimular el reconocimiento social de las funciones de las mujeres y el cumplimiento de sus derechos humanos. En la IV Conferencia Mundial sobre la Mujer (China, 1995), además de estudiar la situación mundial actual de las mujeres, se aprobó, también por consenso, una "Plataforma de Acción" en la que se proponían medidas a aplicar en un plazo de tiempo de quince años, con la finalidad de alcanzar, como ideales, el desarrollo, la igualdad y la paz.

Tal como puede desprenderse de lo sintetizado, los avances conseguidos desde la I Conferencia Mundial hasta la última, son fundamentales, a nivel cuantitativo, pero, especialmente, a nivel cualitativo. Se observa cómo, paulatinamente, los análisis y medidas elaborados se van ampliando incardinándose en metas u objetivos de amplio desarrollo humano. Trabajar por el desarrollo de las mujeres y de las niñas es trabajar por el desarrollo de toda la sociedad. La inversión en la educación y formación de mujeres y niñas conlleva un alto rendimiento en la mejora social. Desde este marco, se puede afirmar que se ha avanzado y trabajado mucho en la relación mujer y derechos humanos, pero que es necesario seguir, continuar y ampliar los esfuerzos a otros ámbitos. En esta línea la creciente, aunque todavía insuficiente, preocupación por la violencia que se comete contra las mujeres, es un indicador de cómo se está avanzando ya por este camino, aunque quede todavía mucho trabajo por hacer y transformaciones sociales por conseguir. Staff (2003), por ejemplo, recoge tres categorías de violación a los derechos humanos de las mujeres: contra su derecho a la integridad personal, a la libertad y a la igualdad. Las violaciones al derecho a la integridad personal, de amplio rango, abarcan desde el maltrato y la violencia doméstica, hasta el homicidio y el uso de la violación como arma de guerra, pasando por la prostitución, la mutilación genital por motivos religiosos o el acoso sexual en el lugar de trabajo. En la categoría de violaciones al derecho a la libertad se incluirían desde el control del cuerpo femenino y de su sexualidad mediante disposiciones legales promulgadas por el estado y la imposibilidad para las mujeres de decidir sin consentimiento o autorización de su pareja masculina, hasta estereotipos y prejuicios culturales que enfatizan la relación de subordinación o de dependencia de las mujeres, limitan sus movimientos y ámbitos de decisión y condicionan su pensamiento y expresión al pensamiento y expresión masculino en el mundo privado o familiar. Las violaciones al derecho a la igualdad suponen la discriminación que sufren las mujeres por su género, al encontrar más trabas en el acceso a los puestos de trabajo, obtener menor remuneración por igual desempeño, sufrir trato desigual en los tribunales de justicia o pasar de víctimas a culpables de la agresión.

Cabe valorar, entonces, las convenciones, declaraciones y planes de acción por lo que de reconocimiento y guía para la práctica y la transformación suponen, y destacar y apoyar todo cambio jurídico, oferta de programas educativos y sensibilizadores, programas sanitarios, realización de estudios e investigaciones..., así como también la labor silenciosa y callada de innumerables grupos de mujeres por el respeto hacia sus derechos y, en general, de todas las personas. Yendo un poco más allá, cuando se trata la cuestión dentro de 
grupos humanos que sufren las consecuencias negativas de otras discriminaciones, el reclamo y la exigencia de cumplimiento de los derechos humanos se vuelve aún más urgente y acuciante. Es lo que sucede en el colectivo de mujeres y niñas gitanas en el que nos vamos a centrar a partir de este momento.

La mujer gitana sufre una triple exclusión: por el hecho de ser mujer, por pertenecer a la comunidad gitan $a^{4} y$ por carecer, en general, de formación académica. En una sociedad donde priman los conocimientos académicos y la acreditación de los mismos -se posean o no-, la comunidad gitana, con un elevado índice de absentismo escolar motivado, entre otros factores, por la no consideración o invisibilidad de la cultura gitana en los contenidos y prácticas habituales en los centros de primaria y secundaria, se sitúa así en situación de mayor exclusión educativa, laboral y social'5. Los ámbitos de relación, familiar, afectivo y social en general, se ven también afectados por esta falta de formación académica y el círculo no sólo no se rompe, sino que se va transformando en una espiral cuya dirección aleja cada vez más a la comunidad gitana del resto de la sociedad.

\section{Brudila Callí: \\ Las Mujeres Gitanas Contra la Exclusión Social}

El equipo de investigación ${ }^{6}$ presentó en diciembre de 2000 un proyecto de trabajo cuyo objetivo principal era contribuir a la superación del fracaso y del absentismo escolar entre las niñas y adolescentes gitanas, contando con la implicación de ellas y de sus familias, para, de esa manera, transformar la situación de exclusión social en que viven gran parte de estas personas en inclusión. El proyecto partía de un supuesto previo: el considerar el sistema educativo formal como herramienta de cambio ante las desigualdades sociales. Asimismo, desde el proyecto se intentaba romper también con ciertos estereotipos acerca de la cultura gitana, por ejemplo, su escasa valoración respecto a los logros académicos y la separación de las niñas a edades tempranas de su ciclo educativo con el fin de prepararlas para el matrimonio, recogiendo, por el contrario, la voz y el sentir de determinadas asociaciones gitanas que reclaman para sus niñas, niños y jóvenes la participación social y la igualdad de condiciones para su formación personal y capacitación profesional. Básicamente, la investigación se planteaba descubrir los factores que producen ese fracaso y absentismo escolar, así como proponer factores superadores de tal situación, realizando ambas metas desde una perspectiva cooperativa, dialógica y basada en la igualdad ${ }^{3}$.

Tres eran los objetivos generales que se perseguían:

1. - Desarrollar teorías y prácticas para superar la exclusión de las niñas y adolescentes del ámbito educativo, contribuyendo al mismo tiempo a solucionar posibles problemas de convivencia.

Este objetivo suponía la definición teórico-práctica de estrategias encaminadas a asegurar el éxito educativo de las niñas y adolescentes, con la finalidad de conferirles mayor autonomía, confianza y seguridad como niñas, adolescentes y futuras mujeres. La igualdad de género y la convivencia intercultural se verían, así, beneficiadas. 
2. Recoger la voz de las mujeres gitanas para identificar y definir los factores transformadores que desde los movimientos y organizaciones de mujeres gitanas se están ya aportando al mundo educativo.

Se parte de considerar a las protagonistas de esta investigación como sujetos activos, de transformación y no de adaptación. Se trata también de partir de lo ya elaborado, de lo ya conseguido y continuar con la tarea; de valorar los logros y avances e intentar alcanzar otros que se concatenen con ellos y nos hagan llegar más lejos.

3. - Elaborar y ofrecer elementos teóricos a cerca de cómo posibilitar el diálogo intercultural.

Considerando las aportaciones sobre aprendizaje dialógico, se pretendía preparar y prepararnos para un diálogo desde la igualdad y el respeto a las diferencias, desde la implicación y el compromiso y tanto dentro de una comunidad como entre comunidades.

Tales objetivos generales, se subdividían en una serie de objetivos específicos que permitían concretar las tareas de análisis y elaboración de alternativas. Se trataba, así, de estudiar los factores causales que llevan a las niñas y adolescentes a abandonar sus estudios y de proponer otros que condujeran a asegurar su continuidad y éxito en su formación. Para ello, su propia identidad, sus necesidades e intereses tenían que hacerse visibles, estar presentes en los currícula educativos y en las tareas escolares. Dado que se parte de considerar a estas personas como sujetos activos de su propia transformación -que no es solamente personal, sino también, y sobre todo, social-, la investigación no podía llevarse a cabo si no se contaba con su total participación e implicación en condiciones de igualdad con la comunidad universitaria. Pero, además, como no se trata tampoco de una situación únicamente personal, sino que tiene un carácter social ineludible -tal como ya hemos planteado-, se necesitaba contar con toda la comunidad, educativa en un primer momento -centro, profesorado, familias- y global -totalidad de agentes sociales presentes en los múltiples marcos de relación-, en segundo término. De esta manera, se puede observar cómo se ampliaban los objetivos del proyecto planteándose llegar a conseguir la convivencia y el diálogo intercultural en condiciones de respeto e igualdad.

Desde este marco, se planteaban tres hipótesis de investigación:

1. - La escuela tradicional privilegia unos perfiles determinados que reproducen la exclusión social derivada de la pertenencia a un grupo cultural minoritario frente a otro que es hegemónico.

La invisibilidad de la cultura gitana es una realidad que ocasiona tanto el temor a perder la propia identidad cultural por parte de la comunidad gitana, como la desvalorización de sus logros y aportaciones culturales que forman parte de la cultura de toda la población española. Como recoge una mujer gitana, estudiante en la Escuela de Trabajo Social: "La cultura gitana no existe en los libros de texto. No se cuenta ni cuándo entran los gitanos en España y eso es parte de nuestra cultura." Tal hipótesis fue confirmada en el transcurso del proyecto. 
2. - Las mujeres gitanas han desarrollado a lo largo de la historia -y continúan haciéndolo- factores transformadores propios.

Esta hipótesis parte de considerar a la persona como creadora de cultura y agente de su propia transformación. Se trata de dar voz a mujeres que, desde sentidos muy diferentes, vienen trabajando para la igualdad social, de género y cultural y, de esta manera, romper estereotipos sociales y culturales. Como expresaba una de las niñas que participaron en la investigación: "Yo soy más gitana que nadie, y muy orgullosa que estoy de ello, y (ello) no significa que no tenga que estudiar (...) y que no tenga inteligencia para estudiar."

3. - Las "buenas prácticas" no se basan en los déficits de los y las participantes, sino en sus competencias como niñas y adolescentes, $y$ como gitanas.

Consideramos necesario basarse en un enfoque caracterizado por las expectativas positivas acerca del logro de los aprendizajes y por la confianza en las capacidades de las alumnas. Partir de las posibilidades, de lo que pueden aportar y aprender, de sus capacidades y habilidades, de sus éxitos y avances, y no de supuestas limitaciones o dificultades. En todo caso, éstas últimas serán las que cualquier niño o niña, en cualquier escuela y en cualquier momento de su ciclo educativo pueden experimentar: "El profesor) dice que hasta que (yo) no aprenda todas las mates y todo, que no se queda tranquilo."

Elemento clave en esta investigación fue el paradigma dentro del que se inscribe, el comunicativo o sociocrítico, encaminado a la transformación de las estructuras sociales. Este paradigma parte de considerar que la realidad se construye históricamente y de forma compartida; aboga por una relación dialéctica entre teoría y práctica; se plantea como finalidades centrales el análisis de esa realidad a la que se concibe como dinámica y susceptible de cambio y de mejora, así como también la autonomía y emancipación de las personas participantes; y defiende la disolución de la frontera entre sujetos de investigación y sujetos investigadores, por la que los sujetos de investigación devienen hacedores y partícipes de la misma. Apoyado, entre otras, por las aportaciones de Habermas y Freire, discurre en un plano de diálogo igualitario en el que las condiciones de validez vienen refrendadas por la comunicación intersubjetiva. Ello supone asegurar que las personas participantes en la investigación dispongan todas de la misma información acerca del contenido y objetivos de la misma, al tiempo que se van consensuando conclusiones, resultados y alternativas.

El proyecto "Brudila Callî" se desarrolló como una investigación longitudinal de 3 años de duración en las Comunidades Autónomas de Aragón, Cataluña y País Vasco. Los cursos académicos en los que se situó fueron, inicialmente, los de $4^{\circ}$ y $5^{\circ}$ de Educación Primaria, considerados como claves para poder estimular el paso a la Secundaria. De hecho, uno de los objetivos de la investigación era, precisamente, estudiar la manera en que se producían cambios de percepción y cambios en la evolución de los resultados académicos de las niñas participantes en la investigación.

Se combinaron técnicas de recogida de información cuantitativas y, especialmente, cualitativas. La primera fase consistió en un primer análisis o aproximación a la realidad, cuyo objetivo era llegar a conocer y concretar la información de la que se dispone, a nivel del estado español, acerca de la escolarización, absentismo y/o fracaso escolar de 
las niñas y adolescentes gitanas -aunque no se excluyó la información relativa a los niños-, en base a fuentes diversas de información, oficiales y no oficiales (como p.e. INE, Departamentos de Educación y Bienestar Social de la Generalitat de Cataluña, CIDE, Secretariado General Gitano, prensa, etc.) . Con esta primera descripción general, y su posterior reflexión crítica, se estaba en mejores condiciones de comenzar a profundizar en los factores, tanto generales como concretos, que inciden en el fracaso escolar de estas futuras mujeres. Paralelamente también se elaboró una parrilla de análisis para el tratamiento de la información recogida mediante técnicas cualitativas. Esta parrilla de análisis consistía en un cuadro de doble entrada, donde recoger referencias al mundo de la vida (familia; cultura gitana; niñas, amigas, mujer); al mundo del sistema (centro educativo; políticas sociales/educativas; cultura y organización de la comunidad gitana; subsistemas -mercado laboral y otros-); y a los espacios de diálogo entre ambos. Las referencias al mundo de la vida, mundo del sistema y espacios de diálogo se categorizaban, a su vez, desde su carácter exclusor -si se referían a trabas u obstáculos para acceder o continuar con los estudios; si ponían en duda sus capacidades para abordar determinadas tareas, etc.- o transformador -si, por el contrario, presentaban la superación de esas barreras o trabas-. En este mismo sentido, las referencias -bien exclusoras o transformadoras- se subdividían en interpretaciones espontáneas -opiniones realizadas de manera directa, espontánea, casi automáticamente-; interpretaciones reflexivas -que suponen un análisis más profundo o argumentado de la opinión manifestada-; teorías -explicaciones generales acerca de algún hecho o rasgo-; e interacciones -opiniones o elaboraciones teóricas elaboradas a partir de la interacción o de procesos sociales-. Todas estas categorías fueron convenientemente definidas con el fin de permitir la univocidad a la hora de analizar la información recogida ${ }^{8}$.

En la misma línea, es interesante destacar la creación de dos Consejos Asesores cuya labor ha sido clave a lo largo de todo el proyecto: uno de niñas y adolescentes gitanas y otro de mujeres gitanas, algunas de las cuales ya colaboraban en otras asociaciones, que han participado en todas las fases del proyecto, aportando conocimientos, colaborando en el diseño de pautas para la recogida de información, participando en el análisis de la misma, consensuando conclusiones, proponiendo alternativas, etc.

Las entrevistas en profundidad, los relatos de vida cotidiana, los grupos de discusión comunicativos y la observación comunicativa, fueron otras técnicas cualitativas de recogida de información. Así, dentro de la primera fase de la investigación, se realizaron y analizaron once entrevistas en profundidad a diferentes personas -niñas, familiares, personas vinculadas a la Administración y profesorado-, con el objetivo de recoger sus percepciones sobre la causa del fracaso escolar y la educación de las niñas gitanas, y conseguir un mejor entendimiento de las mismas. Los guiones de las entrevistas se acomodaron a los diferentes perfiles de las personas entrevistadas y se dieron a conocer tanto el contenido de la entrevista como la finalidad de la investigación y razones por las que habían sido elegidas las personas entrevistadas. En la misma línea, se profundizaba o se volvía a incidir en aquellas respuestas que las personas entrevistadas proporcionaban y que parecían algo dudosas o que podían ser interpretadas en sentidos diferentes, con el objetivo de asegurar la comprensión de la información aportada. Las entrevistas fueron transcritas y analizadas mediante la parrilla de análisis anteriormente comentada 
y fruto de este análisis se pudo elaborar un documento que recoge la voz y percepciones de las personas entrevistadas en torno a las causas del fracaso escolar y la educación de las niñas gitanas.

En la segunda fase de la investigación se procedió a realizar un estudio de casos con el objetivo de conocer y analizar en profundidad situaciones y acciones reales. Este estudio de casos se realizó en seis Centros de Educación Infantil y Primaria de Aragón, Cataluña y País Vasco -dos por cada comunidad autónoma. En cada centro se realizaron dos observaciones -en un aula con niñas gitanas y en una instancia de gestión del centro-; un grupo de discusión o tertulia ${ }^{10}$-con el AMPA (Asociación de Madres y Padres de Alumnos/as) o AFA (Asociación de Familias de Alumnos/as) del centro-; dos entrevistas en profundidad a un miembro del claustro de profesores/as y a un miembro del PAS; y un relato de vida comunicativo a una niña gitana, además de otras informaciones de carácter general proporcionadas por los centros. La observación comunicativa se realiza en situación de determinadas prácticas sociales en las que observadores y participantes comparten y consensúan sus interpretaciones y significados mediante el diálogo igualitario. Por su parte, los grupos de discusión consisten en una tertulia, que se realiza con un grupo natural, en situación espontánea de comunicación, en la que departen acerca de un tema nuevamente desde las bases de diálogo igualitario con el objetivo de validar interpretaciones y asegurar de este modo la comprensión de la información compartida. Finalmente, los relatos de vida pretenden trabajar con la persona a fin de comprender sus opiniones, percepciones, reflexiones, comportamientos e interacciones desde los que construyen sus conocimientos sociales y los aplican en situaciones de la vida cotidiana.

Por último, cabe apuntar también que se realizó una segunda vuelta en el estudio de casos para contrastar la realidad estudiada y corroborar las interpretaciones que se habían elaborado con las personas participantes que viven esa misma realidad. Esta segunda vuelta se realizó en los relatos de vida y los grupos de discusión. Gracias a ella se ha podido profundizar en aquellos temas que parecían destacar o que no habían sido tratados con suficiente detenimiento y se querían continuar y estudiar mejor, sin tener que plantearse la realización de un nuevo grupo de discusión o relato. Igualmente, también se aprovechó para plantear posibles factores transformadores surgidos del análisis de la información/realidad y recoger así la valoración de las personas participantes.

\section{Comunicación de Resultados}

\section{Factores Exclusores y Factores Transformadores}

Como anunciamos en la primera parte de este artículo, hemos analizado los resultados de esta investigación desde una doble perspectiva. En primer lugar, nos interesaba evidenciar aquellos factores que producen el fracaso y el absentismo escolar de las niñas gitanas y, en segundo, descubrir aquellos que a diferencia de los anteriores pueden contribuir al éxito escolar de las mismas. A los primeros los hemos denominado factores exclusores porque barran el paso de las niñas gitanas por el sistema educativo y tienden a excluirlas de las oportunidades y de los beneficios sociales que comporta la educación, 
entre ellos, el acceso a determinados derechos básicos. Los segundos reciben la designación de factores transformadores porque tienen precisamente la capacidad de facilitarnos herramientas para saltar esas barreras y transformar los obstáculos en posibilidades para su superación. Un ejemplo de factor exclusor es la invisibilidad de la cultura gitana en el currículum escolar y las repercusiones que ello comporta en el rendimiento académico de las niñas gitanas. Mientras que el ejemplo contrario, en este caso de tipo transformador, es la inclusión de aspectos de esta cultura en la organización escolar o en los contenidos educativos para favorecer el aprendizaje de las niñas gitanas. De ahí que esta perspectiva de análisis nos permita descubrir los elementos que contribuyen a reproducir las desigualdades educativas y sociales que sufren las niñas gitanas y, simultáneamente, cómo superarlas.

A continuación, vamos a presentar algunos de los factores exclusores hallados más significativos en relación al fracaso y al absentismo escolar de las niñas gitanas. Observaremos a la vez cómo muchos de estos factores impiden que estas niñas puedan disfrutar plenamente del derecho a la educación y, consecuentemente, de otros como son el derecho al trabajo o a la igualdad de géneros. Posteriormente expondremos los factores transformadores que han ido emergiendo a lo largo de la investigación con la finalidad de que puedan servirnos, al mismo tiempo, para orientar acciones educativas encaminadas al éxito escolar de las niñas y adolescentes gitanas.

\section{Factores Exclusores \\ La Cultura Gitana: Una Cultura Estigmatizada}

La comunidad gitana convive con nosotros desde aproximadamente el siglo XV, sin embargo, todavía hoy su cultura continúa siendo la gran olvidada. Los prejuicios sociales y culturales alrededor de esta comunidad han hecho que las distancias entre la cultura gitana y la cultura paya (que es como la comunidad gitana se refiere por oposición a la nuestra) no se hayan reducido significativamente después de tanto tiempo. La imagen social que la cultura hegemónica tiene de la persona gitana es la de una persona indolente, dejada y que no respeta las normas de convivencia. Igualmente, el pueblo gitano se enfrenta a la etiqueta de ser una cultura machista que somete a las mujeres al poder arbitrario de los hombres y que carece de interés por la educación de sus hijos e hijas.

Todos estos prejuicios, como hemos podido comprobar a lo largo de la investigación, impregnan una parte importante de las políticas y prácticas educativas. Y ello explica, a su vez, que las causas del absentismo y el fracaso escolar se atribuyan o bien a la idiosincrasia de la cultura gitana o a sus condiciones de vida. De ahí que a pesar de que las niñas gitanas tienen derecho a acceder a la educación, la falta de expectativas que acostumbran a depositar las administraciones y el profesorado en sus posibilidades educativas y sociales hace que no se busquen las medidas suficientes para atajar el absentismo y el fracaso escolar. Asimismo, según Vega (1997),

"se enmascara la incapacidad del sistema de hacer cumplir la obligatoriedad de la educación escolar que debería disponer de los mecanismos adecuados y pertinentes para que ese precepto legal se cumpla”. 
Por otra parte, como pone de manifiesto una parte del profesorado que entrevistamos, las familias autóctonas cambian a sus hijos e hijas de escuela a medida que la comunidad gitana tiene una presencia mayor en el centro. La razón que exponen habitualmente las familias para explicar esta decisión se relaciona con un supuesto descenso en el rendimiento académico como leemos en el siguiente comentario de una profesora: (...) no quieren que sus hijos estén con los gitanos, (...) porque los ritmos de aprendizaje son más lentos. Esta dinámica está comportando que muchos centros de educación pública a los que asiste población gitana se estén convirtiendo en verdaderos guetos y que algunas escuelas públicas se asocien a la educación de colectivos marginales.

Este hecho es interpretado por las familias gitanas como un signo de racismo y como una de las causas que provoca el desinterés por parte del profesorado en la educación de sus hijos e hijas y en el funcionamiento del centro educativo, como expresa la siguiente madre gitana: (...) el colegio está muy mal desde que hay tantos gitanos, los profesores ha perdido muchisimo interés (...) ¡Hay mucho racismo!.

En resumen, las etiquetas y los prejuicios sobre la comunidad gitana están detrás de muchas actitudes y prácticas exclusoras como son la falta de confianza en las posibilidades educativas de las niñas gitanas y el vaciamiento de los centros educativos de niños y niñas no gitanos con la llegada de esta comunidad a la escuela.

\section{La triple exclusión de la mujer gitana}

La mujer gitana sufre, en general, como decíamos, una triple exclusión: por el hecho de ser mujer, por pertenecer a una minoría étnica subordinada, y por carecer de la formación básica necesaria para acceder al mundo del trabajo y a otros ámbitos de participación social y ciudadana. Además, a ello hay que añadir la imagen estereotipada que la cultura hegemónica proyecta sobre la mujer gitana, contemplando solamente aquellos aspectos de su cultura que la subordinan al hombre, como son la importancia de la virginidad y el matrimonio, y no la percibe en su calidad de sujeto activo. De ahí que no se reconozca el papel que la mujer gitana está desempeñando en el seno de su propia comunidad para transformar sus condiciones de vida y determinadas tradiciones.

Todo ello contrasta, sin embargo, con la percepción que tienen una parte de las mujeres gitanas de sí mismas y de las transformaciones que se están registrando en su propia cultura. Las mujeres gitanas que han participado en esta investigación coinciden a la hora de señalar y valorar los cambios que se están produciendo en su comunidad y el protagonismo cada vez mayor que están adquiriendo como sujetos de cambio y de modernización. En el siguiente fragmento de una tertulia entre mujeres gitanas vemos como la tradición está cambiando en relación a su papel como miembros de su comunidad:

Mujer a: Antes no opinabas nada y ahora opinas.

Mujer b: Ahora tenemos derecho a hablar.

Mujer c: Antes teníamos que esconder la cabeza y lo que diga el marido estaba hecho. Ahora ya no. 
En efecto, en la actualidad, el proceso de modernización reflexiva (Giddens, 1995), supone que las tradiciones se han de argumentar. En la misma línea, Beck (1998, p. 14) señala:

La biografía del ser humano se desliga progresivamente de los modelos y seguridades tradicionales, de los controles ajenos y de las leyes morales generales y, de manera abierta y como tarea, es adjudicada a la acción y a la decisión de cada individuo.

En este sentido, ni la mujer ni la cultura gitanas son una excepción. Las mujeres gitanas ponen de manifiesto que están ganando progresivamente más libertad y, ello, lo atribuyen tanto a su condición de sujetos activos como a las herramientas y a las posibilidades que les da la educación.

Por esta razón, para las mujeres gitanas la educación de sus hijas e hijos es fundamental como expresa de la siguiente manera una madre:

A mi me dicen, y ¡¿cómo fue usted que pensó en meter a sus niñas allí en el colegio? Pues mira, decidí. Yo pensaba ¿estas niñas que van a ser unas analfabetas como yo? Todo el día con un bulto de telas como un perro, casa por casa. Pues si ellas pueden ser algo, pues que no sean como yo. ¡Vamos a variar la vida!

Por otra parte, las formas de subsistencia económica de la comunidad gitana están desapareciendo. Los oficios que han desarrollado tradicionalmente como la recogida de cartón, cristal, chatarra,... y la venta ambulante en los mercadillos no pueden competir en el mercado actual. Por ello, las mujeres gitanas perciben la educación como una oportunidad para superar la exclusión económica y social que sufre el pueblo gitano, especialmente las mujeres. En este sentido, la educación es para la comunidad gitana la puerta a otros derechos básicos como el acceso al mercado de trabajo ${ }^{11}$ y a unas condiciones de vida digna.

El rechazo a la escuela que, en ocasiones, ha podido mostrar la cultura gitana tiene que ver con el miedo a perder su identidad y se relaciona, por tanto, con un tipo de escuela más que con la educación en sí misma. Por ello, una de las reivindicaciones fundamentales desde las organizaciones gitanas es que la escuela incluya aquellos aspectos básicos de su cultura para que las niñas y niños de esta comunidad puedan identificarse con la cultura escolar. Desde esta perspectiva, "está cambiando la idea de desprestigio y apayamiento, por el reconocimiento de la necesidad de la educación. Una educación que tiene un valor en sí misma, que eleva el nivel de formación, sin duda muy importante para nosotros, como un paso previo para conseguir y normalizar una situación social y de trabajo en el futuro de nuestras hijas e hijos" (ASGG, 2000) ${ }^{12}$.

\section{Educación e Identidad Gitana}

Uno de los temores más importantes de la comunidad gitana respecto a la escuela es el de la pérdida de su identidad cultural y los valores que caracterizan a su pueblo como hemos 
introducido en el punto anterior. La invisibilidad de la cultura gitana en el currículum escolar y en la organización del centro educativo hace que las familias desconfíen del tipo de educación que van a recibir sus hijos e hijas. La percepción de que la escuela "apaya" es, en este sentido, un obstáculo para implicar a las familias gitanas en el proceso de educación formal de sus hijas y en el funcionamiento de los centros escolares.

Igualmente, el miedo a ser absorbidos por la cultura hegemónica se extiende a todos aquellos espacios e instituciones en las que la cultura gitana se ha visto tradicionalmente silenciada o excluida. Como leemos en la siguiente reflexión de una mujer gitana:

Cuando dicen ésta está apayada es porque ahora realmente, desgraciadamente para mi pesar, el estudiar y el trabajar lo hacen los payos.

Es decir, el hecho de que el sistema educativo y el sistema laboral se consideren espacios propios de la cultura hegemónica frena el acceso de la comunidad gitana a estos ámbitos de participación social. Y este freno es especialmente grave en el caso de las niñas y mujeres gitanas porque les priva de la posibilidad de estudiar, en muchos casos, más allá de la educación primaria y de encontrar un trabajo fuera del entorno familiar e independizarse económicamente.

En este sentido, una de las reivindicaciones de mayor peso en el terreno de la educación de las mujeres y la comunidad gitana en general, es la del derecho a formarse sin que ello signifique necesariamente la pérdida de sus valores y de sus rasgos de identitad. Algunos de los valores que caracterizan a la comunidad gitana y a los que ésta no desea renunciar los encontramos en la siguiente reflexión de una mujer gitana:

Yo creo que vosotros (refiriéndose a la cultura hegemónica) habéis perdido cosas que nosotros seguimos conservando, porque el respeto a los mayores también vosotros lo habéis tenido (...). La solidaridad entre los gitanos, y sobre todo una cosa muy importante que al igual sí nos diferencia de la gente no gitana sería la importancia de la familia. Le puedes preguntar a catorce mil gitanos y seguramente todos te dirán lo mismo, la familia para los gitanos es lo primero y lo primordial ${ }^{13}$.

El sistema educativo debería reconocer y apreciar estos valores de la comunidad gitana para suavizar el choque entre culturas que viven las estudiantes gitanas. De la misma manera las familias de éstas últimas podrían aproximarse a la comunidad educativa y comprobar que, a veces, el temor percibido a perder su propia identidad puede ser mayor que el real. Establecer puentes entre la cultura escolar y la cultura gitana podría facilitar, como ponen de manifiesto las demandas de las diferentes organizaciones gitanas, la implicación y la complicidad de las familias en el funcionamiento de las instituciones educativas y en la educación de sus hijas. ${ }^{14}$

\section{Algunos Factores Exclusores de la Escuela}

A lo largo de esta investigación hemos visto algunos de los factores exclusores que tienden a barrar el paso de las niñas gitanas por la escuela. De ahí que estos factores nos 
sean de gran utilidad a la hora de explicar las causas que se hallan detrás de unos niveles de absentismo y fracaso escolar tan elevados y, al mismo tiempo, sirvan para encontrar soluciones a esta situación como veremos más adelante. Entre estos factores destacan ahora los siguientes: las bajas expectativas de aprendizaje de las niñas gitanas y el desarrollo de medidas y prácticas educativas segregadoras, el paso de la educación primaria a la secundaria, la ausencia de referentes de la cultura gitana en el entorno escolar y la falta de implicación y de participación de las familias gitanas.

Las bajas expectativas para alcanzar unos resultados académicos deseables y para que las niñas gitanas continúen en el sistema educativo más allá de la educación primaria tienen que ver con el análisis que, en muchas ocasiones, las administraciones educativas y un sector del profesorado hacen sobre las características o condiciones de vida de esta comunidad. Para éstos, las causas del absentismo y del fracaso escolar deben buscarse entre los factores siguientes: la idiosincrasia del pueblo gitano, las condiciones de pobreza extrema en las muchas familias viven o en la falta de motivación e incluso de capacidades del alumnado gitano como podemos leer en la siguiente reflexión de un profesor:

El mayor tópico que se ha tenido, lógicamente en el ámbito de la escuela, que no les interesaba que no ponían interés... que la escuela se les hacía un mundo, que les gustaba esforzarse poco, que son de ritmo más lento, que esto no era lo suyo, que bueno, que estaban más por el baile que por otra cosa...

En este sentido, al atribuir la falta de éxito escolar de las niñas gitanas a sus propias cualidades o a causas externas a las escolares, la clase política, los administradores y el profesorado en general, no priorizan la necesidad de indagar sobre qué responsabilidad tienen ellos en estos resultados y cómo pueden optimizarlos. Esto es así, según Bonal (1997), porque para una parte importante del profesorado la escuela es básicamente una institución neutral. Y dan por supuesto que su trabajo se apoya en principios de igualdad y de justicia social, sin cuestionar discriminaciones que por razón de género, cultura, clase social, etc. se dan cotidianamente en su práctica educativa.

Es precisamente esta falta de expectativas en las posibilidades de aprendizaje de las niñas gitanas la que está detrás de algunas prácticas educativas que tienden a dirigirlas hacia un "currículum de mínimos" o a segregarlas. Nos estamos refiriendo, por ejemplo, al hecho de "bajar el listón" o a segregarlas en aulas y actividades específicas. Lo que suele ocurrir también ante otras situaciones particulares, como alunmando que procede de otras culturas y se incorpora una vez iniciado el curso escolar. Prácticas que suelen reducir los aprendizajes académicos que este alumnado ha de realizar, y ponen el acento en la adquisición de aquellos aprendizajes relacionados con cuestiones comportamentales y con los conocimientos y habilidades que se consideran necesarios para adaptarse a la institución escolar y a la cultura hegemónica. Pero como nos advierten Apple y Beane (2000, p. 29), las escuelas que contienen "estructuras como el encauzamiento y el agrupamiento por capacidades, niegan la igualdad de oportunidades y de resultados a muchos, particularmente a los pobres, a las personas de color y a las mujeres"15. 
Otro de los obstáculos más importantes con el que se encuentran las niñas gitanas dentro del sistema educativo y que va a condicionar su permanencia en éste, se centra en la situación de desventaja con la que enfrentan el paso de la educación primaria a la educación secundaria. Muchas niñas abandonan el sistema educativo justo en el momento que han de dejar la escuela para ir al instituto. Las interpretaciones de los motivos que condicionan esta decisión son de diferente naturaleza. Para una parte importante de los responsables educativos tiene que ver con las tradiciones gitanas, que preparan a las niñas desde muy jóvenes para el matrimonio. Sin embargo, las causas que apuntan los familiares y las mismas niñas son mayoritariamente de otro tipo. Las familias gitanas perciben el instituto como una amenaza para sus hijas. La imagen que tienen de él, a diferencia de la escuela primaria, es la de un centro que funciona sin ningún tipo de regulación, en el que se dan comportamientos violentos, circula la droga y el profesorado no tiene suficiente autoridad para controlar a los estudiantes. En este sentido, como nos dice el siguiente maestro:

(las familias gitanas) no valoran como positivo el ambiente del Instituto. No se sienten seguros, no tienen la seguridad y la tranquilidad que tienen cuando sus hijos están en la escuela, que ha sido una confianza que nos hemos tenido que ganar con el tiempo.

Además de la imagen negativa que muchas familias tienen del instituto, otra dificultad que encuentran para que sus hijas sigan estudiando es la distancia que han de recorrer para asistir a los centros de secundaria. Habitualmente las escuelas o centros de primaria se hallan en la misma zona en la que residen, mientras que para que las niñas acudan a los institutos o centros de secundaria se han de desplazar. Ese desplazamiento y el hecho de que las niñas y adolescentes deban alejarse del territorio o de la zona de confianza de sus familias, se interpreta como una amenaza y se convierte en una barrera en la escolarización de niñas y adolescentes gitanas.

Asimismo, al preguntar a las niñas gitanas qué dificultades o problemas encuentran en el tránsito de la educación primaria a la secundaria apuntan de manera destacada al hecho de que en los institutos no tienen como compañeros y compañeras a miembros de su comunidad o de su propia familia. La falta de referentes y de iguales de su propia cultura tiene un efecto desmotivador que les lleva a interpretar la educación escolar como algo ajeno a ellas.

Por último, la falta de implicación y de participación de las familias gitanas en la vida de los centros educativos supone otro obstáculo, como ha declarado la mayor parte del profesorado que ha sido consultado. Según éstos, las familias gitanas no asisten a las reuniones de padres y madres, no preguntan al profesorado por la evolución académica de sus hijos e hijas y difícilmente colaboran con el profesorado. Sin embargo, al contrastar esta información fundamentalmente con madres gitanas, observamos que una de las explicaciones más plausibles al respecto es la propia imagen que el profesorado tiene sobre la falta de interés de estas familias en la educación de sus hijos/as. Ello provoca que no se busquen los sistemas y canales específicos para incentivar la participación de las 
familias gitanas. Para éstas, sin embargo, las limitaciones más importantes para participar en la escuela y para relacionarse con el profesorado hay que buscarlas en: el horario y en el contenido de las reuniones, en la utilización de un lenguaje excesivamente experto que dificulta el diálogo entre el profesorado y los familiares, y en las relaciones distantes entre unos y otros. Muchas madres gitanas se quejan de que el profesorado sólo las convoca para regañarlas por el mal comportamiento de sus hijos e hijas y, eso hace que se acerquen a la escuela con una cierto temor, desconfianza y desgana.

De ahí que a pesar de que el derecho a la educación es un derecho universal reconocido en la Declaración de Derechos Humanos, en la Constitución Española (1978) y en la Resolución del Consejo y de los Ministros de Educación europeos relativa a la escolarización de los niños gitanos e itinerantes (1989), en la práctica, la discriminación que sufre la comunidad gitana, así como los factores de resistencia y de rechazo de la misma comunidad hacia lo que viene de fuera, impiden que sus miembros puedan disfrutar plenamente de los beneficios que comporta la educación y, a consecuencia de ello, de la oportunidad de acceder a otros derechos básicos como al trabajo y a la vivienda.

\section{Factores Transformadores}

Seguidamente, vamos a presentar algunos de los factores transformadores que surgieron a lo largo de esta investigación y que pueden contribuir a superar las barreras que hemos analizado hasta el momento.

Para que la escuela se configure como un espacio de encuentro y de interacción entre todas las culturas que conviven en un mismo territorio, es importante que las instituciones educativas faciliten el diálogo entre la cultura hegemónica y las otras culturas. De ahí que deban contribuir a visibilizar las aportaciones de los grupos tradicionalmente ignorados y a motivarlos a que hagan uso de su propia voz con la finalidad de que expongan sus necesidades e intereses. En este sentido, hemos visto cómo las reivindicaciones de muchas familias y organizaciones gitanas reclaman una mayor presencia de su historia y de su cultura en el currículum y en determinados aspectos relacionados con la organización del centro:

(...) es necesario promover identidades culturales fuertes y a la vez estilos y formas de interrelación personal y entre culturas basadas en el diálogo y el reconocimiento del otro y de los otros en condiciones de máxima simetría. Para el logro de lo anterior, la aceptación de la diferencia y de lo que nos agrada y no nos agrada de los demás, es decir, la práctica de la tolerancia activa, son objetivos que debemos integrar en todo programa pedagógico y que apela sistemáticamente a cuestiones relativas a los valores y al desarrollo moral de las personas y de los grupos humanos. (Martínez, 1998, p. 89-90).

Pero para que estas transformaciones se den tenemos que encontrar también aquellas soluciones que nos van a permitir que las niñas gitanas permanezcan progresivamente más tiempo en el sistema educativo. De los factores exclusores que hemos planteado en el apartado anterior, se desprenden los siguientes factores transformadores: alto nivel de 
expectativas en las posibilidades de aprendizaje de las niñas gitanas, facilitar el paso de la educación primaria a la secundaria, incluir referentes de la comunidad gitana en el centro educativo, favorecer el acompañamiento mutuo y, por último, incentivar la participación de las familias gitanas en la vida de las instituciones educativas.

Como hemos visto antes, la atribución del absentismo y el fracaso escolar a la falta de capacidades de aprendizaje y de motivación de las niñas gitanas impide que el profesorado introduzca los cambios pedagógicos y metodológicos necesarios para mejorar el rendimiento académico de estas niñas. Para modificar esta situación se requiere, por tanto, conocer y valorar las capacidades e intereses de las niñas y adolescentes gitanas. Como hemos podido observar a través de los diferentes estudios longitudinales que hemos realizado, cuando las niñas gitanas se sienten valoradas y comprenden el sentido que tiene aquello que están aprendiendo, muestran mucho más interés por la escuela y mejoran sus resultados. Igualmente, las metodologías relacionadas con el aprendizaje cooperativo y actividades participativas en el aula estimulan la motivación de las niñas gitanas que acostumbran a realizar la mayor parte sus actividades sociales en grupo o en relación con los suyos. Por esta razón, apartarlas en aulas especiales o distribuirlas en escuelas diferentes para evitar la concentración de población gitana en un mismo centro va en contra de las oportunidades educativas de estas niñas. Se trata, entonces, de promover las interacciones educativas y de incrementar los recursos humanos, tecnológicos, materiales, ... dentro del aula para que no se sientan diferentes y puedan acelerar su proceso de aprendizaje ${ }^{16}$.

Por otra parte, el hecho de que el profesorado parta de unas expectativas altas de aprendizaje contribuye a que se propongan alcanzar los mismos objetivos educativos que con el resto del alumnado. De ahí que la dinámica de "bajar el listón" para reducir los conflictos o las dificultades educativas de las niñas gitanas se substituya por la de incrementar los aprendizajes instrumentales necesarios para que puedan adquirir con mayor facilidad el resto de contenidos educativos. Con ello se evita, asimismo, que las niñas y sus familias desconfíen de la educación porque la consideran una pérdida de tiempo como podemos leer en la siguiente reflexión de un familiar gitano:

El nivel pedagógico (del centro educativo donde llevan a sus hijos e hijas) es el de una guardería de 14 años.

En relación a las barreras que obstaculizan que las niñas gitanas no sigan estudiando al acabar la escuela primaria y que no acudan a los institutos, las conversaciones que hemos mantenido principalmente con las familias nos han servido para plantear algunas propuestas para solucionar esta situación. En primer lugar, parece que un conocimiento mayor de la tarea educativa de los institutos y una imagen más positiva de los mismos daría más confianza a la comunidad gitana. Ello, provocaría que las familias se sintieran más seguras y animarían a sus hijas a seguir estudiando. En este sentido, todas aquellas medidas orientadas a acercar las instituciones educativas a la comunidad gitana y a facilitar el paso de la educación primaria a la secundaria pueden contribuir a generar un clima de confianza y a reducir la tasa de abandono femenino ${ }^{17}$. 
Clima que va a permitir que las familias no perciban este momento de transición de manera tan problemática y como el final, en muchos casos, de la escolarización de sus hijas. En segundo lugar, otro aspecto que facilitaría la continuidad de las niñas gitanas en el sistema educativo es la proximidad del instituto a su lugar de residencia. Las relaciones sociales que establecen entre sí los gitanos y gitanas en el territorio en el que viven hacen que se sientan seguros y no teman por sus hijas. Por último, las niñas gitanas necesitan sentirse acompañadas por miembros de su propia comunidad. La presencia de referentes gitanos (monitores/as, mediadoras/es, profesorado, etc.) y la de otras niñas y niños de su misma cultura son algunos de los factores que las niñas reclaman con mayor insistencia.

En relación a la falta de implicación de las familias gitanas también existen factores que podrían invertir esta situación. Por ejemplo, muchos familiares comentan que se acercan a la escuela cuando reciben un trato de mayor igualdad y las diferencias entre el profesorado y los familiares no están tan marcadas como se desprende del siguiente análisis de una madre gitana:

A nivel incluso de familias con el claustro de profesores, con dirección y todo, ya no somos madres y profesores, esto es una gran familia, no es igual que en otro centro que tienes que pedir por teléfono una cita para poder hablar con el director e igual te dice pues venga dentro de una semana...

De ahí que una organización educativa democrática que promueva la participación y el diálogo entre todos los sectores y culturas que conforman la comunidad educativa tiene capacidad para incentivar, por un lado, la colaboración de las familias gitanas en la educación de sus hijas y, por otro, la implicación de éstas en el funcionamiento de los centros escolares.

Igualmente, consideran necesario que las instituciones educativas sean más sensibles a la idiosincrasia de su comunidad y que la cultura escolar sea capaz de recoger sus aportaciones. Esto sería interpretado por las familias como un signo de acercamiento entre la cultura hegemónica y la cultura gitana, y perderían parte del temor que sienten cuando sus hijas asisten a la escuela como expresa un padre gitano:

Claro que queremos que estudien, pero arriesgamos una de las formas de guardar nuestra cultura.

En este sentido, la presencia de la cultura gitana en el currículum y en los órganos de decisión de los centros educativos puede contribuir a que se sientan acogidos y a que perciban la escuela como un espacio propio.

\section{La Voz de las Niñas Gitanas}

Hemos dejado para un lugar preferente la voz de las niñas gitanas para tratar de reflejar cómo perciben la educación y cuáles son sus expectativas de futuro. Como hemos indicado ya en otros apartados, una parte importante del profesorado piensa que las niñas gitanas no están suficientemente motivadas para cursar con éxito sus estudios debido a 
aspectos de su cultura, del ambiente en el que viven o a características personales. Sin embargo, las niñas nos aportan una visión algo diferente. Para ellas, una de las razones que explica la falta de interés que en muchas ocasiones sienten por los estudios tiene que ver con la sensación de que están perdiendo el tiempo. Piensan que o bien no aprenden lo suficiente o que los aprendizajes que realizan son irrelevantes. Igualmente acusan al mal ambiente escolar que se vive en algunos centros educativos de su falta de interés para ir al instituto, como podemos leer en los siguientes fragmentos de entrevistas con diferentes niñas gitanas:

E: ¿Por qué dejaste el instituto?

Niña1: Porque no me gustaba y porque aprendía a hacer el gamberro, y porque no tenía tiempo.

Niña2: Cuando iba a la Primaria me gustaba más que no dónde estoy ahora (instituto) porque estudiaba mucho. Pero cuando pasé a la ESO (educación secundaria obligatoria) fatal.

En general, hemos podido observar cómo la valoración que las niñas hacen de los centros de primaria es significativamente más positiva que la de los institutos. Las causas que éstas señalan son desde la calidad y utilidad de los aprendizajes que realizan, el clima escolar y la proximidad del profesorado. Por otra parte, en los centros de primaria es más fácil que coincidan con otros niños y niñas de su familia y de su cultura y que el profesorado se muestre más sensible a las diferencias.

Respecto a las expectativas de futuro de las niñas que han sido consultadas, la mayoría coincide en la voluntad y en las ganas de trabajar cuando sean mayores. Y a diferencia de sus madres y abuelas ya no ven el mercadillo o el trabajo familiar como la única opción. Una parte importante de las niñas al preguntarles: -¿qué quieres ser de mayor?-, respondían: -peluqueras-. Para las niñas gitanas la peluquería es una meta alcanzable que no les exige muchos estudios y que les va a dar independencia económica y personal. Sin embargo, cuando en el curso de las entrevistas y de las conversaciones con ellas se ampliaba el abanico de posibilidades mostraban un gran interés por ámbitos profesionales como la medicina, la abogacía, el magisterio, etc. De ahí que, seguramente, si esas opciones no se las habían planteado nunca antes sea debido a la falta de expectativas que la comunidad educativa y la sociedad en general deposita sobre las capacidades y las posibilidades de las niñas y mujeres gitanas. Por esta razón, pensamos que una de las responsabilidades principales que el sistema educativo y el profesorado deben contraer con las niñas gitanas y en general con todos aquellos colectivos que tradicionalmente han sido silenciados y excluídos, es la de transmitirles la confianza necesaria para que puedan superar con éxito cualquier barrera y abrirles el horizonte más amplio posible, de forma que pronto puedan verse realizados sueños como el que expresó la siguiente madre gitana:

Dentro de 100 años lo que tengo muy claro es que me encantaría que hubieran médicos gitanos, abogados gitanos, ique ya los hay! Pero que eso, que los niños gitanos fueran al cole, o sea sin perder lo que somos pero teniendo lo que tenéis todos. 


\section{Conclusiones}

Hace aproximadamente 52 años que la Declaración Universal de Derechos Humanos reconoció los derechos y libertades fundamentales de todas las personas, sin que se pueda hacer distinción alguna por motivos de raza, sexo, idioma o religión. Desde entonces la comunidad internacional ha desarrollado múltiples mecanismos para promover y proteger los derechos humanos. Sin embargo, todavía hoy, muchas mujeres ven como sus derechos más básicos son objeto constante de numerosas violaciones. Está situación es aún más grave en el caso de las mujeres que pertenecen a minorías étnicas subordinas y de aquellas que se han visto excluidas sistemáticamente de la educación formal. En este sentido, la especial situación de la mujer gitana hace que sea uno de los colectivos que con más fuerza percibe el rechazo social y ve vulnerados su derechos. Igualmente, la situación escolar de las niñas y adolescentes gitanas que hemos analizado en la investigación que relatamos en este artículo es un ejemplo más del camino que todavía nos queda por recorrer para alcanzar la igualdad de oportunidades y la realización universal de los derechos humanos.

Tanto la "Convención sobre la Eliminación de Todas las Formas de Discriminación contra la Mujer" (1979) como la "Convención sobre la Eliminación de Todas las Formas de Discriminación Racial” (1969), ponen de manifiesto el papel central que tiene la educación para acabar con el sexismo y el racismo y para promover el reconocimiento de la diversidad. La escuela es, sin duda, mucho menos sexista y racista que otras instituciones sociales, y su evolución está marcada por un carácter profundamente inclusor y democrático. No obstante, todavía hoy, quizá por falta de concienciación, tiende a privilegiar la cultura hegemónica frente a las subordinadas y no reconoce suficientemente las aportaciones y las competencias de los colectivos tradicionalmente excluídos, como planteábamos en las hipótesis de esta investigación refiriéndonos a la situación de la mujer gitana.

El objetivo de esta investigación no ha sido sólo, sin embargo, conocer aquellos factores que tienden a reproducir las discriminaciones sexuales y racistas en la escuela o en otras instituciones sociales, sino descubrir al mismo tiempo aquellos otros que contribuyen a su superación. De ahí que una parte fundamental de la investigación y de este artículo se haya centrado en la definición de los factores exclusores -aquellos que tienden a barrar el éxito académico y la continuidad de la niñas gitanas en el sistema educativo-, y de los factores transformadores que a diferencia de los anteriores reducen la tasa de abandono de las niñas gitanas de los estudios y promueven el aprendizaje.

Los prejuicios sobre la cultura gitana, la falta de reconocimiento del papel que desempeñan las mujeres gitanas de esta comunidad, la exclusión económica que padecen y el bajo nivel de instrucción de la comunidad gitana son algunos de los factores que están contribuyendo en mayor grado a perpetuar la situación de exclusión social, económica, política, cultural,... del pueblo gitano. Todo ello nos ayuda explicar también los elevados índices de absentismo y de fracaso escolar. La invisibilidad de la cultura gitana en el currículum, la falta de expectativas de los administradores y profesionales de la educación, las prácticas segregadoras y la falta de confianza entre familias y edu- 
cadores, son algunos de los factores exclusores propios de la institución escolar que impiden que muchas niñas gitanas puedan disfrutar plenamente de los derechos humanos. Trabajando desde la colaboración e implicación de un sector y otro se conseguirá hacer real el derecho de todas las mujeres a la educación.

En contraposición a los factores anteriores, queremos acabar señalando algunos de los factores transformadores que pueden contribuir a cambiar esta situación y que esperamos puedan servir para orientar estrategias para acabar con la discriminación de la mujer gitana. Algunos de estos factores son: la inclusión de la cultura y la voz de las mujeres gitanas en el currículum y en la organización del centro, las altas expectativas de aprendizaje a partir del reconocimiento de las capacidades de las niñas gitanas y la colaboración entre las familias, el profesorado y la administración para asegurar que el paso de la educación primaria a la secundaria se produce sin deserciones por parte de las niñas gitanas.

La correspondencia entre educación y equidad es la piedra angular de cualquier sociedad democrática que aspire a la realización de los derechos humanos. Por esta razón, pensamos que la educación de las niñas gitanas es la mejor garantía para que puedan participar, en condiciones de igualdad, en la vida política, económica y cultural. de las sociedades de las que forman parte, y para que puedan contribuir a edificar una sociedad cada vez más justa e inclusiva.

En esta línea, una última reflexión, la realización de los derechos humanos depende en buena medida, como se desprende de todos tratados, acuerdos, normativas internacionales, de la educación. Una educación que ha de incluir la formación en derechos humanos y que, bajo nuestro punto de vista, se ha de desarrollar en el seno de instituciones profundamente inclusivas y democráticas; es decir, en instituciones en las que la observancia a los derechos humanos y el respeto a las libertades fundamentales se constituyan en principios rectores.

\section{Notas}

1. Fuente: Amnistía Internacional-Sección Española.

2. "Las estrategias de Nairobi orientadas hacia el futuro para el adelanto de las mujeres hasta el año 2000”.

3. Queda pendiente, sin embargo, otra reclamación: la relativa al sesgo androcentrista del contenido de la misma Declaración. En palabras de Staff (2003). “(...) esos derechos humanos sólo han tenido como referencia al sexo masculino, que se considera como paradigma de lo humano, sin que se tomara en cuenta la manera de sentir, pensar, luchar y vivir del sexo femenino, que representa la otra parte de la humanidad, pero que ha sido invisibilizada y se le niega el reconocimiento de sus derechos específicos y simplemente se la incluye como formando parte integrante del hombre."

4. La comunidad gitana, india de origen y europea y transnacional por movilidad, llegó a Europa a mediados del siglo XIV. Su llegada a España fue, inicialmente, bien recibida. Sin embargo, la época de intolerancia y represión que se inició con la unificación de los Reyes Católicos (siglo XV) supuso el origen de la discriminación que esta comunidad sobrellevará hasta nuestros días. La Constitución Española (1978) parecía augurar un futuro de igualdad y convivencia que, finalmente, no se ha hecho realidad. Podríamos caracterizar la cultura gitana desde los siguientes rasgos: pueblo sin territorio; de tradición 
ágrafa u oral; con lengua propia, el romanò; con una Ley Gitana no escrita, pero aceptada por todos los miembros de la comunidad que se convierten en agentes de su respeto; y con un amplio cuerpo de tradiciones, costumbres y valores, como el respeto a las personas mayores, la hospitalidad y solidaridad entre los miembros de la comunidad, el cumplimiento de la palabra dada o su especial aprecio por la libertad, entre otros elementos.

5. Las estadísticas sobre la comunidad gitana son escasas y no resulta tampoco fácil acceder a las existentes. Según datos de la Unión Romaní (Unión del Pueblo Gitano), organización reconocida por las Naciones Unidas, la comunidad gitana en 1998 se acercaba a los 12.000.000 de personas en Europa, siendo Rumanía el país con mayor población gitana. La tasa de analfabetismo entre sus miembros oscilaría entre el 33\% y el 60\%; el índice de desempleo sería elevado; y la mayoría de la población viviría todavía en zonas y barrios marginales, con bajo índice de salubridad. La proporción de estudiantes universitarios y personas licenciadas, si bien comienza a apuntar, se mantendría aún en niveles bajos. Por otra parte, siguiendo a López Escudero (2001), a nivel del estado español, el absentismo radical afectaría a un 10\% del alumnado gitano; un 35\% presentaría absentismo escolar esporádico (a veces motivado por cuestiones laborales de la familia); el abandono al iniciar la ESO se acercaría al 30\%; y los índices generales de fracaso escolar se situarían entre un 60 y un $70 \%$.

6. La información que vamos a recoger a partir de este momento es fruto del Proyecto de Investigación I+D+I del Plan Nacional MTAS-Instituto de la Mujer, otorgado en diciembre de 2000 y concluido en diciembre de 2003, cuyo equipo oficial estaba formado por: M. Payá (investigadora principal), A. Ayuste, E. Oliver, L. Puigvert y R. Valls (Universidad de Barcelona); S. Miguel y S. Sánchez (Universidad Ramón Llull); C. Elboj y Ma J. Vicen (Universidad de Zaragoza); D. Padua (Universidad de Almería); M. Arandia (Universidad de Bilbao); y S. Giménez (Programa "Acceder" de Huelva). Esta investigación se realizó en el contexto de trabajo de CREA (Centro Especial de Investigación en Teorías y Prácticas superadoras de las desigualdades. Universidad de Barcelona).

7. El planteamiento de la investigación y los resultados de las diferentes etapas se pueden consultar en la siguiente página web: http://www.pcb.ub.es/crea/Brudila/index.html

8. La parrilla de análisis y la definición de las categorías se puede consultar en la siguiente página web: http://www.pcb.ub.es/crea/Brudila/Metodologia.html

9. Ello supuso definir previamente los perfiles de las personas entrevistadas.

10. Cabe recoger la excepción de un CEIP de Aragón donde la ténica del grupo de discusión fue substituida por una entrevista en profundidad a una madre de la comunidad gitana

11. A esta forma de igualdad se refiere el artículo 11 de la "Convención sobre la Eliminación de Todas las Formas de Discriminación contra la Mujer”.

12. En esta línea, en las III Jornadas sobre la situación de la mujer gitana: formación y empleo organizadas por la Asociación de Mujeres Romaní, celebradas en Granada (España) el 16 de Mayo de 1992 se formularon las siguientes reivindicaciones: a) las mujeres gitanas queremos trabajar y vemos en el trabajo un medio de promoción, de colaboración familiar y autonomía personal; b) las mujeres gitanas necesitamos poder elegir nuestra formación y nuestro trabajo; c) la mayor dificultad es la escasa formación y un cierto rechazo a la hora de buscar trabajo por ser gitanas; d) los oficios tradicionales gitanos no tienen salida, preferimos un trabajo normalizado, bien asalariado o autónomo, donde tengamos un reconocimiento social; e) nos parece importante que los poderes públicos financien y apoyen la formación de la mujer gitana; f) nos gustaría que los hombres gitanos entendieran, apoyaran y facilitaran nuestra promoción y nuestro movimiento asociativo.

13. Otros valores del pueblo gitano son: el cuidado de los hijos y de los ancianos que gozan del respeto y la consideración máxima, la hospitalidad, el cumplimiento de la palabra dada, el sentido de la libertad como condición natural de la persona, el sentido de la solidaridad 
para con los miembros de la etnia como obligación y el cumplimiento de las decisiones tomadas por los mayores cuando éstos las tomas según la Ley Gitana (Vega, A. 1997), en http://www.unionromani.org (consultada el 28/09/2004).

14. En ocasiones, se pudiera pensar que el interés y la preocupación de determinadas políticas educativas y de algunos centros por favorecer la integración cultural, no incluye la comunidad gitana.

15. De ahí que este tipo de prácticas no facilite la superación de la discriminación contra la mujer gitana y, por extensión, la de todos aquellos colectivos que el sistema educativo tiende a excluir o a dirigir hacia los itinerarios de "segunda categoría". Por ello, conviene que los Estados parte recuerden el compromiso a adoptar medidas como la que se recoge en el apartado a) del artículo 10 de la "Convención sobre la eliminación de todas las formas de discriminación contra la mujer": Acceso a los mismos programas de estudios, a personal docente del mismo nivel profesional y a locales y equipos de la misma calidad.

16. Existen experiencias educativas que llevan muchos años trabajando en esta línea pedagógica y que han desarrollado sistemas de organización y metodologías muy apropiadas para tratar la diversidad. Es el caso, por ejemplo, de las Escuelas Inclusivas (http://www.inclusiveschools.org/), las Escuelas Aceleradas (http://www.accelerated.org) y las Comunidades de Aprendizaje (http://www.comunidadesdeaprendizaje.net/).

17. Como se propone en el apartado f) del artículo 10 de la "Convención sobre la eliminación de todas las formas de discriminación contra la mujer".

\section{Referencias}

Acker, S. (1995). Género y educación. Madrid: Nárcea.

Apple, M. W. y Beane, J. A. (1999). Escuelas democráticas. Madrid: Morata.

ASGG. (2000). El pueblo gitano y la educación. En ASGG, Gitanos, pensamiento y cultura, 7/8, Diciembre 2000, pp. 30. Madrid: ASGG.

Ayuste, A.; Flecha, R.; López, F.; Lleras, J. (1998). Planteamientos de la pedagogía crítica. Comunicar y transformar. Barcelona: Graó.

Beck, U. (1998). El normal Caos del Amor. Barcelona: El Roure.

Bonal, X. (1997). Las actitudes del profesorado ante la coeducación. Barcelona: Graó.

Geertz, C. (1996). Los usos de la diversidad. Barcelona: Paidós-ICE/UAB.

Giddens, A. (1995). Modernidad e identidad del yo. Barcelona: Ediciones Península.

Gil, F.; Jover, G.; Reyero, D. (2001). La enseñanza de los derechos humanos. Barcelona: Paidós. Guttman, A. (2001). La educación democrática. Barcelona: Paidós.

Habermas, J. (1999). La inclusión del otro. Barcelona: Paidós.

Jordán, J. (1994). La escuela multicultural. Un reto para el profesorado. Barcelona: Paidós. Juliano, D. (1993). Educación Intercultural. Madrid: Eudema.

Levin, H. M.(1998). Accelerated Schools: a decade of evolution. En A. Hargreaves, A. Lieberman, M. Fullan, D. Hopkins (Eds.), International Handbook of educational change Part two (pp. 807-830). Norwell: Kluwer Academic Publishers.

López Escudero, A. (2001). La escolarización de los gitanos. El Pais, 22 de octubre. Lorde, A. (2003). La hermana, la extranjera. Madrid: Horas y horas.

Martínez, M. (1998). El contrato moral del profesorado. Bilbao: Desclée de Brouwer.

Payà, M. (1997). Educación en valores para una sociedad abierta y plural: Aproximación conceptual. Bilbao: Desclée De Brouwer.

San Román, T. (1976). Vecinos gitanos. Madrid: Akal.

Staff, M.(2003) Mujer y derechos humanos. http://www.derechos.org/koaga/viii/staff.html (última consulta: 28 de septiembre de 2004). 
Taylor, C. (1993). El multiculturalismo y la "política del reconocimiento". México: Fondo de Cultura Económica.

Touraine, A. (1997). ¿Podremos vivir juntos? Iguales y diferentes. Madrid: PPC.

Trilla, J. (1992). El profesor y los valores controvertidos. Barcelona: Paidós.

Vega, A. Los gitanos en España. http://www.unionromani.org/histo.htm (última consulta: 28 de septiembre de 2004).

VV.AA. Pueblo gitano. http://www.unionromani.org/pueblo_es.htm (última consulta:

28 de septiembre de 2004).

VV.AA. (2003). Comunidades de aprendizaje. Transformar la educación. Barcelona: Graó. 